\title{
Abundancia y diversidad del fitoplancton en el Embalse de Salto Grande (Argentina - Uruguay). Ciclo estacional y distribución espacial
}

\author{
Lizet De León ${ }^{1}$ y Guillermo Chalar ${ }^{2}$
}

Sección Limnología, Facultad de Ciencias, Universidad de la República. Iguá 4225 CP: 11400. Montevideo

- Uruguay. 1- e-mail: lizetdl@fcien.edu.uy; 2- e-mail: gchalar@fcien.edu.uy

\section{RESUMEN}

El embalse de Salto Grande es un ecosistema artificial creado para la producción hidroeléctrica. Este sistema tiene un tiempo de residencia bajo, 12.3 días, su régimen térmico es polimíctico y la morfología es dendrítica. En este embalse se ha estudiado la composición, abundancia y diversidad del fitoplancton en relación con el ciclo estacional y los parámetros físicos y quími-

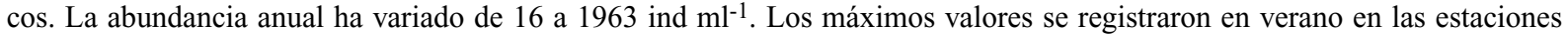
situadas en brazos con dominancia de Aulacoseira y Microcystis. Los valores mínimos se encontraron en el canal principal y durante el invierno donde dominaban los fitoflagelados nanoplanctónicos. La diversidad anual mostró un patrón inverso con un rango de variación entre 0.6 y 4.4 bits ind $^{-1}$. En este trabajo se discute una sucesión estacional de estrategas C a R y S, como sugiere Reynolds 1988.

Palabras clave: fitoplancton, diversidad, sucesión de estrategas, embalses.

\begin{abstract}
Salto Grande reservoir is an artificial ecosystem created for hydroelectric power generation. This system has low retention time, 12.3 days, polymictic regime, and dendritric morphology. The composition, abundance and diversity of phytoplankton in relation with the seasonal cycle and physicochemical parameters were investigated in this reservoir. Annual abundance varied from 16 to 1963 ind $\mathrm{ml}^{-1}$. The maximum values were registered in summer, in the stations located in the branches with Aulacoseira and Microcystis dominance. The minimum ones corresponded to the main channel in winter were nanoplanktonic phytoflagellates dominated. The annual diversity showed an inverse trend ranging between 0.6 and 4.4 bits ind $^{-1}$. In this paper, a succession from C to R and S strategy is discussed, as suggested by Reynolds (1988).
\end{abstract}

Keywords: phytoplankton, diversity, strategist succession, reservoirs.

\section{INTRODUCCIÓN}

El embalse de Salto Grande constituye una obra de represamiento del Río Uruguay de carácter binacional Uruguayo-Argentino. Fue creado para generación de energía hidroeléctrica entrando en operación en 1979. Es utilizado además para abastecimiento de agua potable, navegación, riego y recreación. Según Salas y Martino (1990), de acuerdo a su temperatura media anual mayor a $15^{\circ} \mathrm{C}$ y su temperatura mínima superior a $10^{\circ} \mathrm{C}$, el sistema podría considerarse como cálido tropical. En base a las características físico-químicas, el embalse está formado por dos subsistemas definidos por el cauce principal y los brazos laterales (Berón, 1990; Chalar et al. 1993; Conde et al, 1996) y presenta características de ambiente meso-eutrófico. La comunidad fitoplanctónica está dominada por diatomeas del género Melosira (Aulacoseira spp), en casi todo el sistema y durante gran parte del año, siendo acompanadas por cyanobacterias Microcystis aeruginosa en los brazos Gualeguaycito y Mandisoví (margen argentino), al final del verano y otoño (Quirós y Luchini, 1983).

Este estudio pretende aportar información nueva sobre la composición y dinámica de la comunidad fitoplanctónica y analizar su distribución espacial y temporal, en relación a los parámetros abióticos. 
Tabla 1. Características morfométricas del Embalse Salto Grande. Morphometric features of the Salto Grande Reservoir.

\begin{tabular}{lc}
\hline Superficie & $780 \mathrm{~km}^{2}$ \\
Volumen & $5109 \times 10^{6} \mathrm{~m}^{3}$ \\
Profundidad media & $6.4 \mathrm{~m}$ \\
Profundidad máxima & $35.0 \mathrm{~m}$ \\
Ancho medio & $5.4 \mathrm{~km}$ \\
Ancho máximo & $9.0 \mathrm{~km}$ \\
Longitud & $100 \mathrm{~km}$ \\
Desarrollo línea de costa & 11.9 \\
Perímetro & $1190 \mathrm{~km}$ \\
Tiempo de residencia promedio & 11.3 días \\
\hline
\end{tabular}

\section{ÁREA DE ESTUdIO}

Las características morfométricas del Embalse de Salto Grande se detallan en la tabla 1. El Río Uruguay es el principal tributario. El mismo nace aproximadamente a $60 \mathrm{~km}$ del Océano Atlántico en la Serra do Mar (Santa Catarina Brasil) y recorre $1800 \mathrm{~km}$ en dirección Oeste y Sur, hasta su desembocadura en el Río de la Plata. Su lecho es principalmente de roca basáltica, siendo el margen uruguayo más elevado que el argentino. El régimen hidrológico del río presenta sus mayores caudales durante el invierno y los menores en verano. El caudal medio en

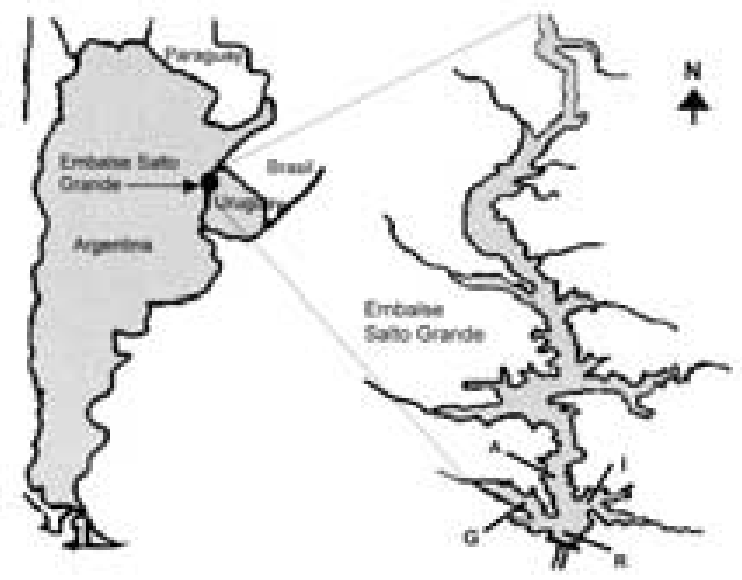

Figura 1. Esquema mostrando la ubicación del Embalse de Salto Grande en la región y las estaciones de muestreo en el embalse. R- Represa, A- La Paloma, G- Gualeguaycito e IItapebí. Diagram showing Salto Grande Reservoir location in the region, and the sampling stations in the reservoir. $R$ Dam, A-La Paloma, G- Gualeguaycito e I-Itapebí.
72 años de registro fue de $4643 \mathrm{~m}^{3} \mathrm{~s}^{-1}$ con mínimos y máximos de 92 y $36100 \mathrm{~m}^{3} \mathrm{~s}^{-1}$ respectivamente (CTM, 1982).

La cuenca del Río Uruguay abarca 297199 km² de extensión en territorios de Brasil, Argentina y Uruguay, mientras que la cuenca del embalse es de $224000 \mathrm{~km}^{2}$. Las precipitaciones anuales en la cuenca tributaria varían entre 1000 y $2000 \mathrm{~mm}$. En la cuenca del embalse se registra un promedio anual de $1260 \mathrm{~mm}$. Las temperaturas medias anuales son de $19^{\circ} \mathrm{C}$ y los vientos predominantes son del NE y SO con promedios anuales de 7 y $14 \mathrm{~km} \mathrm{~h}^{-1}$ respectivamente.

\section{MATERIAL Y MÉTODOS}

Se seleccionaron cuatro estaciones de muestreo en la cabecera del embalse, dos en el curso principal denominadas Represa (R) y La Paloma (A), una en el brazo Gualeguaycito $(\mathrm{G})$ en el margen argentino, al Este, y otra en el brazo Itapebí (I) en el margen uruguayo, al Oeste (Fig. 1). Las muestras se tomaron con una frecuencia bimensual entre mayo y setiembre (invierno) y mensual entre setiembre y abril (primavera a otoño).

En cada estación se midió la temperatura y se tomaron muestras para la determinación de sólidos suspendidos totales, fósforo total, fosfato, nitratos y silicatos, según la metodología descrita en Conde et al. (1997). Se tomaron muestras para el análisis cualitativo de fitoplancton mediante arrastre superficial con una red de plancton de 33 $\mu \mathrm{m}$ y para el análisis cuantitativo y de clorofila $a$ con bomba de succión a las profundidades correspondientes al $100 \%, 50 \%, 1 \%$ de luz incidente en la superficie de la columna de agua y a $1 \mathrm{~m}, 5 \mathrm{~m}$ y $10 \mathrm{~m}$ por debajo del límite del $1 \%$. Esta última profundidad no fue considerada para la clorofila $a$. Las muestras cualitativas se fijaron in situ con formalina neutralizada al $4 \%$ de concentración final. Para la clasificación de las Divisiones se siguió el criterio de Silva (1972, en Parra y Bicudo, 1995). Los organismos flagelados nanoplanctónicos $(<30 \mu \mathrm{m})$ no identificados se agruparon bajo la denominación de "Fitoflagelados". Para el análisis cuantitativo, las 

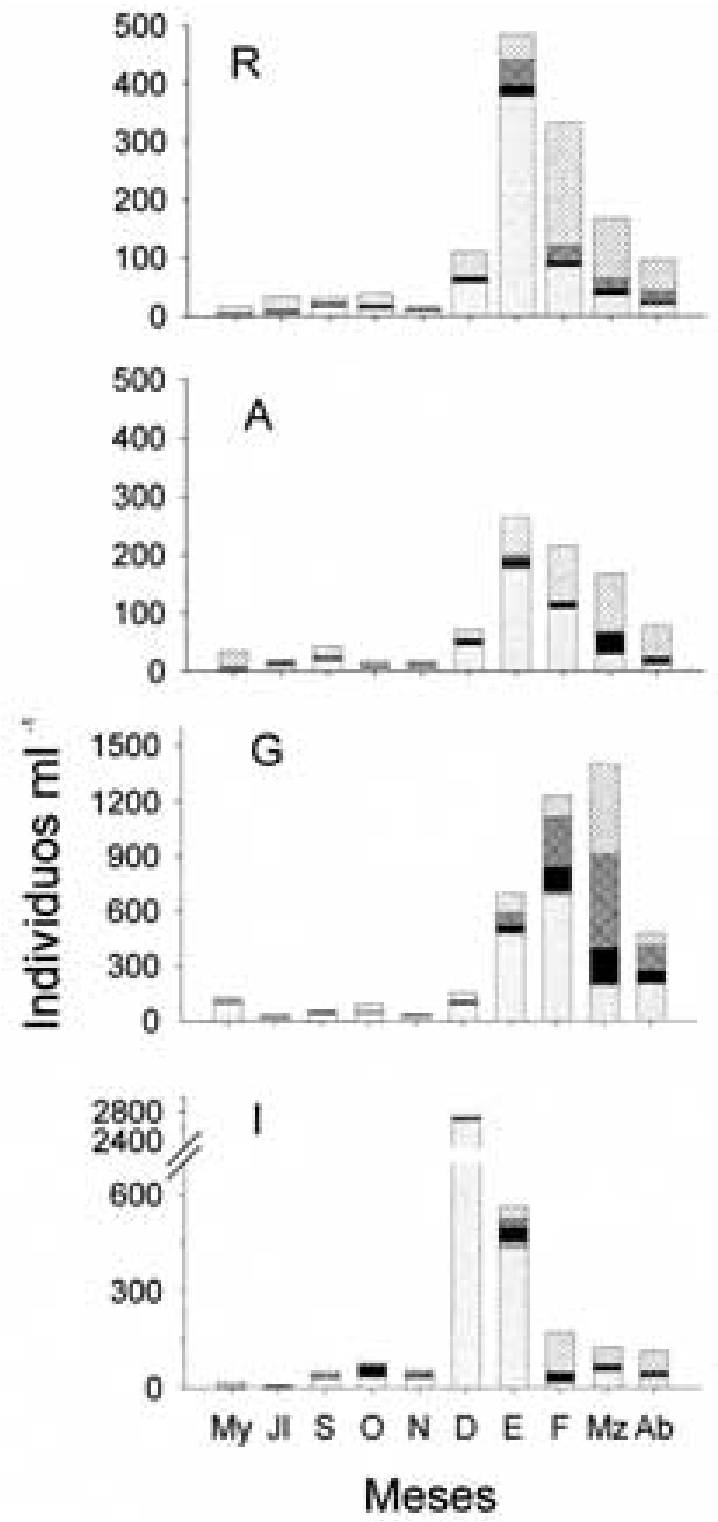

एma Aulacoseira spp, $\square$ Navicula sp, Anabaena spp, XXXX Raphidiopsis mediterranea,

Figura 2. Variación anual de la abundancia de los taxa dominantes en las estaciones de muestreo. Annual variation of the abundance of phytoplankton dominant taxa at the sampling stations.

muestras se fijaron in situ con solución lugol y se realizó el recuento con microscopio invertido utilizando cámaras de sedimentación de 2, 5, 10, 20 y $50 \mathrm{ml}$ (según Utermöhl, en Hasle, 1978). Las especies se cuantificaron por organismos, consi- derándose como tal una colonia, un filamento o una célula, según el nivel de organización específico. Se definieron como especies abundantes aquellas que registraron una abundancia relativa mensual igual o mayor al $5 \%$ en, al menos, un mes y en dos estaciones como mínimo; especies frecuentes las que registraron abundancia relativa mensual igual o mayor al $0.5 \%$ en un mes y dos estaciones y especies raras las que registraron abundancia relativa mensual menor al $0.5 \%$. El análisis de clorofila $a$ se realizó con etanol caliente (Nush, 1980) y se midió la absorbancia a 665 y $750 \mathrm{~nm}$ con un espectrofotómetro (UV-V).

\section{RESULTADOS}

\section{Composición taxonómica}

Se determinaron 187 taxa correspondientes a las Divisiones Chlorophyta (105), Bacillariophyta (25), Euglenophyta (21), Cyanophyta (20), Chrysophyta (7), Dinophyta (4), Cryptophyta (3) y Xanthophyta (2). Del total de taxa registrados, 19 correspondieron a nuevas citas para Uruguay (De León y Pérez, en preparación).

\section{Variación espacial y temporal del fitoplancton}

La variación anual de la comunidad fitoplanctónica se analizó a partir de las especies más abundantes. Del total de 187 taxa determinados, 25 resultaron abundantes. La comunidad del fitoplancton del Embalse Salto Grande, estuvo compuesta por un bajo número de especies abundantes $(14.4 \%$ ) y un número alto de especies raras $(53 \%)$. Las especies frecuentes representaron el $47 \%$ del total de taxa determinados.

La abundancia de la comunidad fitoplanctónica, analizada en base a las especies frecuentes (Fig. 2), presentó un rango de variación entre 16 org. $\mathrm{ml}^{-1}$ ( estación $\mathrm{R}$, mayo) y 1963 org. $\mathrm{ml}^{-1}$ (I, diciembre). La distribución vertical de los organismos determinó mayor abundancia en la zona eufótica, mientras que espacialmente la mayor abundancia se registró en las estaciones de los 


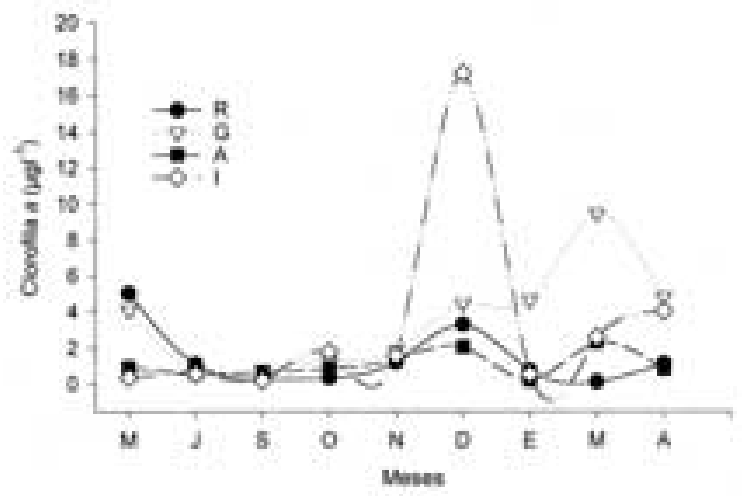

Figura 3. Variación anual de la clorofila $a$ en superficie, en las estaciones de muestreo. Annual variation of surface chlorophyll $a$ at the sampling stations.

brazos y temporalmente en verano (entre diciembre y abril). En las estaciones R y A (cauce principal), las abundancias promedio anuales fueron similares entre sí y menores que en G e I (brazos).

La comunidad fitoplanctónica registró su menor abundancia $\left(<100\right.$ ind $\left.\mathrm{ml}^{-1}\right)$, entre mayo y noviembre en las cuatro estaciones de muestreo. En diciembre se incrementó la densidad de organismos principalmente en I (ca. 2000 org. $\mathrm{ml}^{-1}$ ), mientras que en enero, el incremento se registró en todas las estaciones. Estas diferencias se debieron principalmente al crecimiento de Bacillariophyta en I (en diciembre) y de Cyanophyta en G (febrero, marzo y abril), seguidas de Bacillariophyta y flagelados (Fig. $2)$. Dentro de estos grupos las especies más abundantes fueron: Aulacoseira sp y A. ambigua en I y Aulacoseira sp, A. ambigua, Anabaena planctonica y Raphidiopsis mediterranea en G.

\section{Biomasa del fitoplancton}

La biomasa fitoplanctónica, medida como la concentración de clorofila $a$, registró un valor promedio anual de $2.01 \mu \mathrm{g}^{-1}$ para las 4 estaciones, 5 profundidades y 9 meses analizados. Los menores valores se registraron en las estaciones del cauce principal ( $\mathrm{R}$ y $\mathrm{A})$, con un mínimo de $0.15 \mu \mathrm{g}^{-1}$ en mayo (Fig. 3). La mayor concentración de clorofila $a$ se registró en diciembre, con un máximo de $17.2 \mu \mathrm{g}^{-1}$ (promedio para la

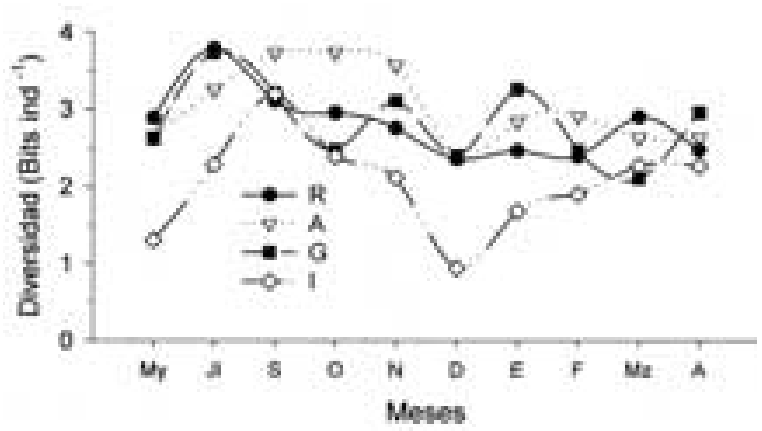

Figura 4. Variación anual de la diversidad fitoplanctónica en las estaciones de muestreo. Annual variation of phytoplankton diversity at the sampling stations.

columna de agua) en I. Dicho aumento se encontró asociado a un incremento de la abundancia de Aulacoseira spp. En G la mayor concentración se registró en marzo y abril $\left(7.0 \mu \mathrm{g} \mathrm{1^{-1 }}\right.$ y $5.7 \mu \mathrm{g} \mathrm{l}^{-1}$, respectivamente) y estuvo asociada al aumento de las poblaciones de Cyanophyta (Anabaena planctonica, Raphidiopsis mediterranea).

\section{Diversidad del fitoplancton}

Para el análisis de diversidad se utilizaron los datos de abundancia de las especies más frecuentes. Se excluyeron los fitoflagelados por tratarse de un grupo multiespecífico.

Los valores de diversidad oscilaron entre 0.6 y 4.4 bits ind $^{-1}$ con un promedio de 2.68 para todo el sistema. Los valores más altos correspondieron generalmente a los meses fríos (mayo a octubre) mientras que la menor diversidad fue registrada en los meses cálidos (diciembre, febrero y marzo) (Fig. 4).

En las estaciones R y A, el índice de diversidad fue mayor al comienzo del período de estudio, disminuyendo progresivamente. G e I presentaron la misma tendencia en el tiempo, pero con mayores oscilaciones. En diciembre se registró una disminución de la diversidad en todas las estaciones y en enero un incremento.

\section{Parámetros ambientales}

La temperatura mínima del agua se registró en julio $\left(11.8{ }^{\circ} \mathrm{C}\right)$, aumentando hasta alcanzar el 
máximo en enero $\left(26.5{ }^{\circ} \mathrm{C}\right)$. No se registraron diferencias verticales que permitieran definir una estratificación térmica. El caudal de ingreso (Qi) y el caudal de salida (Qs) fueron altos durante el invierno y menores en verano (Fig. $5)$. Durante los meses de verano se registró un aumento del nivel del agua del embalse de aproximadamente $1 \mathrm{~m}$. Consecuentemente con las variaciones de los aportes y salidas de agua y el manejo del nivel de la cota, el tiempo de residencia del embalse fue mayor en verano con un máximo de 24 días en diciembre y menor en invierno con un mínimo de 4 días en julio (media anual $=14$ días). La temperatura del agua se correlacionó positivamente con el tiempo de residencia $(p<0.01)$ (Fig. 6A). La precipitación local medida en la estación meteorológica cercana al área de estudio presentó un comportamiento similar al tiempo de residencia, mostrando que la magnitud de dichos aportes no fueron significativos comparados con los aportes provenientes de la cuenca media y alta aportados por el cauce principal.

La concentración de sólidos suspendidos, fosfatos y fósforo total presentaron los valores máximos en invierno y se correlacionaron positivamente entre sí $(p<0.01)$ y negativamente con el tiempo de residencia $(\mathrm{p}<0.01)$ (Fig. 6B). La concentración de nitratos y silicatos (Fig. 6C), presentó un comportamiento diferente de los

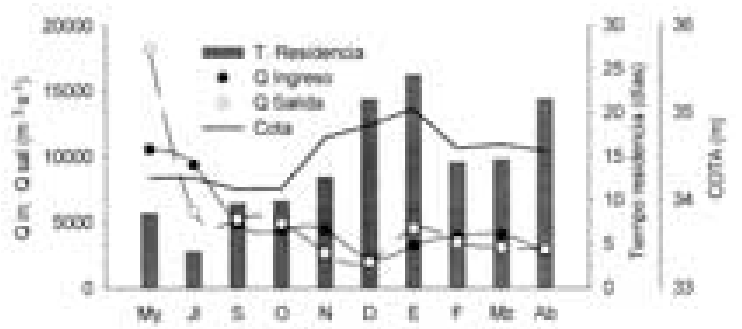

Figura 5. Variación anual de los parámetros físicos y químicos (media de las cuatro estaciones). A- temperatura y tiempo de residencia. B- Sólidos suspendidos totales (SST), fosfatos $\left(\mathrm{PO}_{4}\right)$ y fósforo total (PT). C- Precipitación local, nitratos $\left(\mathrm{NO}^{2-}{ }_{3}\right)$ y silicatos $\left(\mathrm{SiO}^{3-}{ }_{2}\right)$. Annual variation of the physical and chemical parameters (mean of the four sampling stations). A-temperature and retention time. B-Total suspended solids (SST), phosphates $\left(\mathrm{PO}_{4}\right)$ and total phosphorus (PT). C-Local rainfall, nitrates $\left(\mathrm{NO}^{2-}{ }_{3}\right)$ and silicates $\left(\mathrm{SiO}^{3-}{ }_{2}\right)$. parámetros anteriores, con máximos en los meses de verano, relacionándose con el aumento de la precipitación local.

\section{DISCUSIÓN}

\section{Composición y ciclo estacional}

La comunidad fitoplanctóncia del embalse estuvo dominada por Bacillariophyta, Cyanophyta y fitoflagelados nanoplanctónicos. Junto con dichos grupos se determinaron una importante variedad de especies de Chlorophyta y Euglenophyta y pocas especies de Chrysophyta, Cryptophyta y Dinophyta. Esta composición fue similar a la encontrada por Quirós y Lucchini (1983), en el mismo sistema y por Bonilla (1997) y Pérez et al. (1999), en los embalses sobre el Río Negro (Uruguay). La variedad de especies de Chlorophyta, Bacillariophyta y Euglenophyta, coincidió con los resultados encontrados por O'Farrell e Izaguirre (1994), referidos a la composición del fitoplancton del Río Uruguay. Espacialmente, se registraron diferencias entre la composición taxonómica del cauce principal y los brazos, principalmente debido a la dominancia de diatomeas en las primeras y la mayor densidad de cianobacterias en las últimas. Entre las especies de Aulacoseira, fue muy frecuente y abundante la especie $A$. ambigua y su forma spiralis. Esta especie no fue encontrada en otros ríos de la región, constituyendo un taxa característico del Río Uruguay (O’Farrell, 1994).

Según estudios anteriores (O'Farrell, 1994; O'Farrell e Izaguirre, 1994 y O'Farrell et al., 1996), realizados en el Río Uruguay y en quince importantes ríos de la cuenca del Río de la Plata incluyendo diversos tramos del Río Paraná y afluentes, la composición de la comunidad fitoplanctónica del Embalse de Salto Grande puede definirse como eupotamoplanctónica.

De acuerdo con Reynolds (1988), la comunidad fitoplanctónica se estructura, básicamente, en relación a la disponibilidad de nutrientes y luz. En ambientes con disponibilidad de nutrientes, predominan organismos oportunis- 


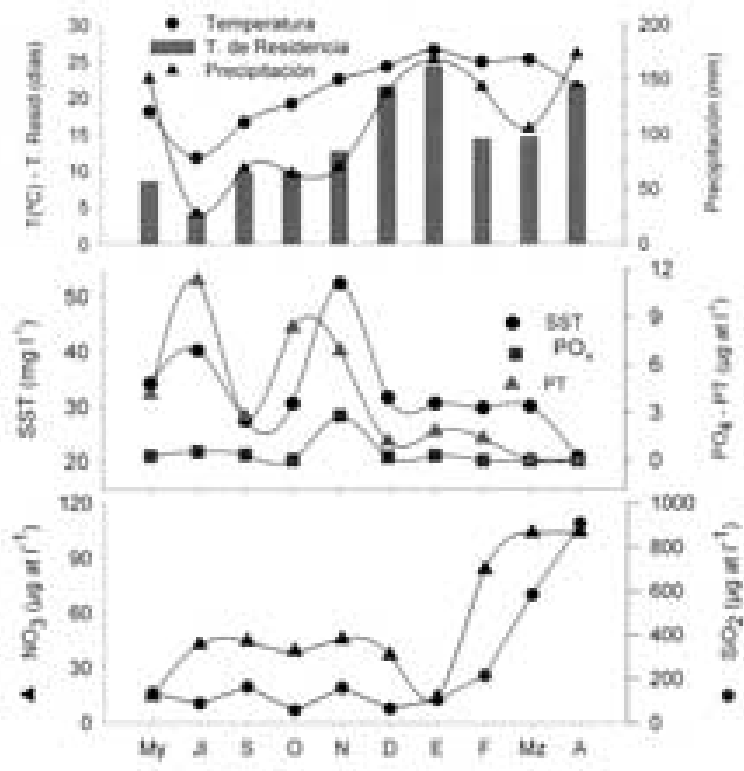

Figura 6. Variación anual del tiempo de residencia hidráulico, caudales (Q) de ingreso y salida y de la cota en el Embalse de Salto Grande. Annual variation of the retention time, inflow (Q Ingreso), outflow (Q Salida), and elevation at Salto Grande Reservoir.

tas, de rápido crecimiento y reproducción, generalmente de pequeño tamaño y alta relación superficie/volumen, denominadas C-estrategas. En ambientes con limitación de nutrientes, predominan los organismos tolerantes a este stress o S-estrategas. En ambientes con cambios frecuentes en la calidad de luz debido generalmente a turbulencias, predominan los R-estrategas. De Melo y Húszar (2000), encontraron predominancia de organismos de pequeño tamaño y características de $\mathrm{C}$ estrategas al comienzo del período de mezcla en el Lago Batata, Brasil . Según estos autores, las condiciones de flujo permanente favorecen el desarrollo de estos organismos sobre otros grupos. La presencia cuantitativamente dominante de los fitoflagelados nanoplanctónicos en el Embalse de Salto Grande al comienzo y final de este estudio, podría explicarse por sus características de organismos oportunistas, alta relación superficie/volumen, alta eficiencia para la incorporación de nutrientes y alta tasa de reproducción
(Reynolds, 1984, 1988; Sandgren, 1988). Ello los coloca en el grupo de los C estrategas. El mayor desarrollo de las cianobacterias coincidió con el período de concentración indetectable de fósforo y con la mayor disponibilidad de nitrógeno y silicatos. Ello pondría en evidencia su eficiencia para crecer en ambientes limitados por nutrientes, especialmente $\mathrm{P}$ (Reynolds, 1988, 1997), representando a los $\mathrm{S}$ estrategas. La dominancia de formas filamentosas sobre las coloniales, indicaron las condiciones turbulentas del sistema, característica que también fue encontrada por Hino (1979), para el embalse de Lobo (Broa). Microcystis aeruginosa, la especie colonial más abundante, alcanzó mayor desarrollo en $\mathrm{G}$, mostrando las características más eutróficas de esta estación.

Las diatomeas céntricas del género Aulacoseira dominaron la comunidad fitoplanctónica del Embalse Salto Grande, por su densidad y presencia constante en la mayoría de las muestras. Resultados similares fueron observados en estudios anteriores de este sistema (Quirós y Luchini, 1983; Berón, 1990), en otros embalses de la región (Bonilla, 1997; Gómez, 1990, 1991; Hino, 1979; O'Farrell, 1994; Pérez et al., 1999; Reynolds et al., 1986; Tundisi, 1990a; Vila, 1987) y en los embalses españoles (Margalef et al., 1976; Planas, 1975). Estos organismos, característicos de sistemas turbulentos y sometidos a constantes cambios en la calidad de luz debido a sus movimientos a través del gradiente lumínico, son típicos $\mathrm{R}$ estrategas. La dominancia de Aulacoseira, indicaría que el embalse Salto Grande tiene características de un gran río (Gómez, 1990, 1991; O’Farrell et al., 1996), especialmente en su cauce central.

Durante el período de estudio se observó una sustitución de los estrategas C-S-R, en respuesta a las variaciones del ambiente (Fig. 7). En otoño e invierno (mayo y julio), cuando se registraron los mayores caudales de ingreso y las mayores concentraciones de nutrientes y sólidos suspendidos, se encontró una alta abundancia de los fitoflagelados nanoplanctónicos. Nuestros resultados indican que la baja temperatura, la alta turbulencia y la poca penetración de la luz debida a 
la alta turbidez inorgánica, habrían limitado la presencia de otras especies (Gliwitz, 1999; Reynolds, 1984, 1997; Sommer, 1993), favoreciendo a las más eficientes en la incorporación de nutrientes, con alta tasa de crecimiento (estrategas C) y con mayor eficiencia fotosintética para desarrollarse en un ambiente turbio y turbulento (estrategas R). A finales de invierno y en primavera (setiembre a diciembre) la disponibilidad de nutrientes y el incremento de la radiación solar y la temperatura favorecieron el crecimiento de las Chlorophyta de pequeño tamaño (C-estrategas). En noviembre, coincidiendo con una alta concentración de $\mathrm{SiO}_{2}$ y el máximo de $\mathrm{PO}_{4}$ y SST, se registró un aumento de la abundancia de las Bacillariophyta (R-estrategas). El máximo poblacional alcanzado en diciembre, se reflejó en el incremento de la concentración de clorofila $a$ y en la disminución de los nutrientes al final de la primavera y verano (diciembre a marzo). Desde febrero hasta abril, la concentración de $\mathrm{P}$ fue indetectable, determinando la presencia de las Cyanophyta, tolerantes al stress de nutrientes o S-estrategas. A partir de febrero dis- minuye la abundancia de las Bacillariophyta y aumenta la población de fitoflagelados (C-estrategas), indicando el comienzo de un nuevo ciclo a partir de otoño. Los resultados indican una presencia de estrategas $\mathrm{C}$ en otoño e invierno, sustituidas por $\mathrm{R}$ en primavera y verano y éstas por $\mathrm{S}$ al final del verano y otoño.

\section{Abundancia, biomasa y diversidad}

La abundancia del fitoplancton del embalse presentó dos períodos claramente diferenciados: 1abril a noviembre (invierno) y 2- diciembre a marzo (verano). Durante el primer período, la

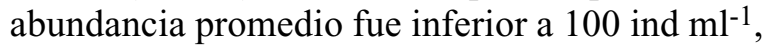
similar a la registrada para lagos oligotróficos (Izaguirre et al., 1990), de régimen hidrológico variable (García, 1980) y sistemas lóticos (García y Anselmi, 1989; Zalocar y Vallejos, 1982) de la misma región. En cambio, la abundancia registrada a partir de diciembre, superior a 1000 ind $\mathrm{ml}^{-1}$, estuvo en el rango de los ambientes lénticos (García y Anselmi, 1989) mesotróficos a eutróficos (Calijuri y Tundisi,

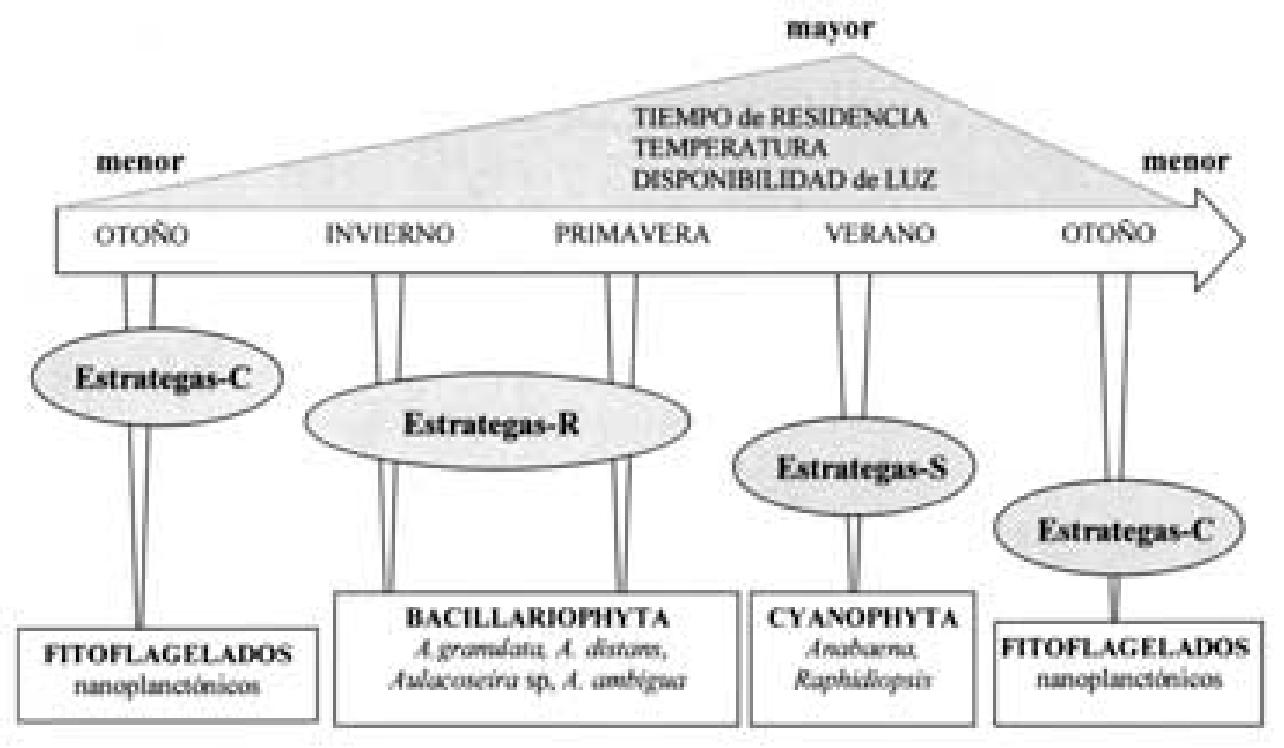

Figura 7. Sucesión estacional de los estrategas fitoplanctónicos C, S y R y su relación con los factores ambientales en el Embalse Salto Grande. Seasonal succession of phytoplanktonic $C, S$ and $R$ strategists, and its relationship with environmental factors at the Salto Grande Reservoir. 
1990; García, 1977; Izaguirre y Vinocur, 1994a; Izaguirre et al., 1990) o de grandes ríos (O’Farrell y Izaguirre, 1994; Schiaffino, 1981; Zalocar y Vallejos, 1982).

La biomasa fitoplanctónica medida como concentración de clorofila $a$ fue baja. Según la escala utilizada por Tundisi et al. (1988), en base al índice de Carlson para 23 embalses del Estado de Sao Paulo, el sistema se clasificaría como ultraoligotrófico a oligotrófico (concentración promedio menor a $2.5 \mu \mathrm{g}^{1^{-1}}$ ). Según el índice utilizado por Salas y Martino (1990) para sistemas cálidos tropicales, Salto Grande sería oligotrófico (62\% de probabilidad (Chalar et al., 1993). El patrón de variación temporal de la biomasa fitoplanctónica fue igual al registrado por Montecino y Cabrera (1982), para el embalse Rapel, un sistema eutrófico monomíctico templado y por Bonetto et al. (1972) para embalses de la región subtropical templada.

La diversidad del fitoplancton de Salto Grande fue relativamente baja (2.98 bits ind ${ }^{-1}$ promedio, $0.5-4.1$ bits ind $^{-1}$ ), en relación a los valores encontrados por O'Farrell y Izaguirre (1994), para el Río Uruguay aguas arriba (4.15.8 bits ind $^{-1}$ ) y abajo del embalse (3.2-4.3 bits ind $^{-1}$ ). Estos valores indican la alteración de las condiciones ambientales del río al pasar de un régimen fluvial a un embalse (Tundisi 1983, 1990b), pero no pueden considerarse per se indicadoras de determinado estado trófico. Izaguirre et al. (1990), determinaron un rango de 0.67 a 4.51 bits ind $^{-1}$ en 20 cuerpos de agua ultra-oligo a eutróficos, de la región andino patagónica de Argentina. Sin embargo, no pudieron establecer diferencias de nivel trófico en base al valor de diversidad. Lobo y Kobayasi (1990), encontraron mayor diversidad en ambientes con mayor índice de saprobiedad, cuando esperaban lo contrario. Los mejores indicadores de cambios en las características tróficas de ríos y arroyos serían las variaciones en la composición y abundancia del fitoplancton, más que los índices de diversidad (Stoermer y Smol, 1999).

En Salto Grande, los valores más altos de diversidad se registraron en invierno, cuando la densidad fitoplanctónica fue baja $\left(<100 \mathrm{ind} \mathrm{ml}^{-1}\right)$ y el sistema presentó características lóticas. Los menores valores se registraron en el verano, en los brazos cuando se observaron las mayores densidades de organismos $\left(>1000\right.$ ind $\left.\mathrm{ml}^{-1}\right)$ y el sistema presentó características más lénticas. Las comunidades sometidas a disturbios frecuentes de mediana intensidad presentan diversidad alta (Holzman, 1993; Pádisak, 1993; Reynolds, 1993; Sommer, 1993; Sommer et al., 1993). Esto indicaría que el régimen lótico del embalse constituye un disturbio frecuente para la comunidad, probablemente debido a los pulsos provocados por los aportes de nutrientes, materiales particulados y la turbulencia generada en el sistema. Izaguirre y Vinocur (1994b) encontraron valores de diversidad entre 4.0 y 4.8 en ambientes lénticos de la región con baja densidad de organismos (690 a 16500 ind $\mathrm{ml}^{-1}$ ), coincidiendo con Branco y Senna (1996), que encontraron correlación negativa entre densidad y diversidad. Según Margalef (1997), la disminución de la diversidad indica un incremento de la actividad metabólica de los organismos, de modo que las variaciones de diversidad están asociadas a las características productivas de la comunidad. La marcada estacionalidad de la abundancia y biomasa del fitoplancton y de las características ambientales estudiadas, serían los factores determinantes de las diferencias temporales en la diversidad encontradas en este sistema.

\section{Estado Trófico}

En la caracterízación trófica de los cuerpos de agua se utilizan diversos indicadores entre los cuales se encuentran la composición, abundancia, biomasa y diversidad fitoplanctónica. La composición del Embalse Salto Grande estuvo generalmente dominada por diatomeas de ambientes eutróficos, coincidiendo con los resultados de Gómez (1991) en el Embalse del Rio III. También fue similar a la composición de los embalses eutróficos analizados por Bonetto et al. (1976) y a las asociaciones eutróficas y mesotróficas determinadas por O'Farrell e Izaguirre (1994b). Según Duarte et al. (1992), los lagos dominados por diatomeas tendrían 
características mesotróficas, mientras que los dominados por cianobacterias serían eutróficos (Lampert y Sommer, 1997; Margalef, 1983; Paerl, 1988; Pizzolón 1996; Scheffer et al., 1997). En Salto Grande, las cianobacterias fueron más abundantes en los brazos, especialmente en G. Berón (1990) y Quirós y Lucchini (1983), mencionaron la presencia de floraciones de $M$ aeruginosa, prácticamente uniespecífica, entre abril y diciembre de 1980 en G. La densidad de $M$. aeruginosa registrada en este estudio fue alta pero no alcanzó a los valores reportados por estos autores.

\section{AGRADECIMIENTOS}

Este trabajo fue financiado por el Convenio entre la Comisión Técnica Mixta de Salto Grande y la Facultad de Ciencias, Universidad de la República Oriental del Uruguay. Los autores desean agradecer a los compañeros de la Sec. Limnología por su colaboración en los muestreos y análisis químicos, a la Lic. Ma. del Carmen Pérez por su asistencia en la identificación taxonómica del fitoplancton, al Lic. Alejandro Otaegui, Dto. de Ecología de la CTM, por su apoyo a esta investigación y al Dr. Oscar Parra, Centro EULA de la Universidad de ConcepciónChile, por sus sugerencias.

\section{BIBLIOGRAFÍA}

BERON, L. 1990. Features of the limnological behavior of Salto Grande's reservoir (ArgentinaUruguay). Ecological Modelling, 52: 87-102.

BONILLA, S. 1997. Composición y abundancia fitoplanctónica de tres embalses en cadena sobre el Río Negro, Uruguay. Iheringia, ser. Bot., 49:47-61.

BONETO, A., D. DI PERSIA, R. MAGLIANESI y M. CORIGLIANO. 1976. Caracteres limnológicos de algunos lagos eutróficos de embalse de la región central de Argentina. Ecosur, 3(5): 47-120.

BRANCO, C. y P. SENNA. 1996. Phytoplankton composition, community structure and seasonal changes in a tropical reservoir (Paranoa Reservoir, Brazil). Algological Studies, 81: 69-84.
CALIJURI, M. C. y J. G. TUNDISI. 1990. Limnologia comparada das Rs do Lobo (BROA) e Barra Bonita. Estado de Sâo Paulo. Mecanismos de funcionamento e bases para o gerenciamento. Rev. Brasil. Biol., 50 (4): 893-913.

CHALAR, G., L. DE LEON, R. DE LEON, D. FABIAN y J. GORGA. 1993. Evaluación de la eutrofización del Embalse de Salto Grande. Análisis de las relaciones entre los parámetros físico-químicos y biológicos. Informe Final de la primer etapa. CTM-SG/Universidad de la República-Facultad de Ciencias-Sección Limnología.

CONDE, D., W. PINTOS, J. GORGA, R. DE LEON, G. CHALAR y R. SOMMARUGA. 1997. The main factors inducing chemical spatial heterogeneity in the Salto Grande, a reservoir on the Uruguay River. Arch. Hydrobiol. Suppl. 113. Large Rivers, 10 (1-4): 571-578.

DE MELO, S. y V. HUSZAR. 2000. Phytoplankton in an amazonian flood-plain lake (Lago Batata, Brazil): diel variation and species strategies. $J$. Plankton. Res., 22(1): 63-76.

DUARTE, C. M., S. AGUSTÍ y D. CANFIELD. 1992. Patterns in phytoplankton community structure in Florida Lakes. Limnol. \& Oceanogr., 37(1): 155-161.

GARCÍA, M. O. 1980. Fitoplancton de una laguna del valle aluvial del Paraná medio ("Los Matadores", Santa Fe, Argentina). I. Estructura y distribución en relación a factores ambientales. Ecología, Argentina, 4: 127-140.

GARCÍA, M. O. 1977. Ciclo anual del fitoplancton en el Embalse San Roque (Córdoba, Argentina). Rev. Asoc. Cienc. Nat. Litoral, 8: 1-12.

GARCÍA, M. O. y M. I. ANSELI. 1989. Fitoplancton y variables ambientales en la cuenca del Río Saladillo (Sta. Fe, Argentina). Rev. Brasil. Biol., 49(4): 957-967.

GLIWICZ, Z. M. 1999. Predictability of seasonal and diel evens in tropical ans temperate lakes and reservoirs. En: Theoretical Reservoir ecology and its Applications. J. G. Tundisi \& M. Straskaba (eds.): 99-124. International Institute of Ecology, Brazilian Academy of Sciences and Bachuys Publishers.

GOMEZ, N. 1990. Variaciones morfológicas de Aulacoseira granulata (Ehrenberg.) Simonsen (Bacillariophyceae) en el embalse Río III, Córdoba, Argentina. Biota, Chile, 6: 45-51.

GOMEZ, N. 1991. Poblaciones de diatomeas planctónicas en una $\mathrm{R}$ subtropical: Embalse Río III, Argentina. Annls. Limnol., 27(1): 3-14. 
HASLE, F., 1978. Counting phytoplankton. En : Phytoplankton Manual. Monographs in Oceanographic Methodology. Sournia, A. (ed.). UNESCO, 6: 88 - 96.

HINO, K. 1979. Analise qualitativa e quantitativa do microfitoplancton da $R$ do Lobo (BROA) Sâo Carlos, Sâo Paulo. Tese de Maestrado. Univ. Federal de Sâo Carlos-SP-Brasil.

HOLZMAN, R. 1993. Seasonal fluctuations in the diversity and compositional stability of phytoplankton communities in small lakes in upper Bavaria. Hydrobiol., 249: 101-109.

IZAGUIRRE, I. y A. VINOCUR. 1994a. Typology of shallow lakes of the Salado River Basin (Argentina), based on phytoplankton communiities. Hydrobiol., 277:49-62

IZAGUIRRE, I. y A. VINOCUR. 1994b. Algal assemblages from shallow lakes of the Salado River Basin (Argentina). Hydrobiol., 289:57-64

IZAGUIRRE, I., del GIORGIO, I. O'FARRELL y G. TELL. 1990. Clasificación de 20 cuerpos de agua andino-patagónicos (Argenitna) en base a la estructura del fitoplancton estival. Cryptogamie, Algol., 11: 31-46.

LAMPERT, W. y U. SOMMER. 1997. Limnoecology. Oxford University Press.

LOBO, E. y H. KOBAYASI. 1990. Shannon's Diversity Index applied to some diatoms assemblages in the Sakawa River system (Kanagawa Pref., Japan) and its use as an indicator of water quality. Jpn. J. Phycol. (Sôrvi), 38: 229-243.

MARGALEF, R. 1997. Our Biosphere. En: Excellence in Ecology $n^{o}$ 10. O. Kinne (Ed.).. Ecology Institute - Germany.

MARGALEF, R., D. PLANAS, J. ARMENGOL, A. VIDAL, N. PRAT, A. GUISET, J. TOJA y M. ESTRADA. 1976. Limnología de los embalses españoles. Ministerio de Obras Públicas. Madrid.

MARGALEF, R. 1983. Limnología. Omega.

MONTECINO, V. y S. CABRERA. 1982. Phytoplankton activity and standing crop in an impoundment of central Chile. J. Plankton Res., 4(4).

NUSCH, E.A. 1980. Comparison of different methods for chlorophyll and phaeopigments determination. Arch. Hydrobiol. Beih. Ergebn. Limnol., 17:14-36.

O'FARRELL, I. 1994. Comparative analysis of the phytoplankton of fifteen lowland fluvial systems of the River Plate Basin (Argentina). Hydrobiol., 289: 109-117.
O'FARRELL, I., I. IZAGUIRRE y A. VINOCUR. 1996. Phytoplankton ecology of the Lower Paraná River (Argentina). Arch. Hydrobiol. Suppl., 115/Large Rivers, 11 (1): 75-89.

O'FARRELL, I y I. IZAGUIRRE. 1994. Phytoplankton ecology and limnology of a River Uruguay Lower Basin (Argentina). Arch. Hydrobiol./Suppl., 99 (Monographische Beiträge) (1/2): 155-179.

PAERL, H. 1988. Growth and reproductive strategies of freshwater blue-green algae (cianobacteria). En: Growth and reproductive strategies of freshwater phytoplankton. Sandgren C. (ed.). Cambridge University Press.

PARRA, O. y C. BICUDO. 1995. Introducción a la biología y sistemática de las algas de aguas continentales- Univ. De Concepción.

PÉREZ, M. C.; S. BONILLA y G. MARTÍNEZ. 1997. Phytoplankton community of a polymictic reservoir, La Plata River Basin, Uruguay. Rev. Brasil. Biol., 59(4): 353-541.

PIZZOLON, L. 1996. Importancia de las cianobacterias como factor potencial de toxicidad en las aguas continentales. Interciencia, 21: 239-245.

PLANAS, D. 1975. Distribution and productivity of the phytoplankton in Spanish reservoirs. Verh. Internat. Verein. Limnol., 19: 1860-1870.

QUIRÓS, R y L. LUCHINI. 1983. Características limnológicas del embalse de Salto Grande III: Fitoplancton y su relación con parámetros ambientales. Rev. Asoc. Cienc. Nat. Litoral, 13: 19-66.

REYNOLDS, C. S., 1997. Vegetations processes in the pelagic: a model for eccosystem theory. En: Excellence in Ecology $n^{\circ}$ 9. Kinne, O. (Ed.). Ecology Institute, Germany.

REYNOLDS, C. S., 1993. Scales of disturbance and their importance in plankton ecology. Hydrobiol., 249: 157-171.

REYNOLDS, C. S., 1988. Functional morphology and adaptative strategies of freshwater phytoplankton. En: Growth and reproductive strategies of freshwater phytoplankton. Sandgren C. (Ed.). Cambridge University Press.

REYNOLDS, C. S. 1984. The ecology of freshwater phytoplankton. Cambridge University Press.

REYNOLDS, C.; V. MONTECINO; M. GRAF y S. CABRERA. 1986. Short-term dynamics of a Melosira populations in the plankton of an impoundment in Central Chile. J. Plankton Res., 8(4): 715-740. 
SANDGREN C. (Ed.). 1988. Ecology and reproductive strategies of freshwater phytoplankton. Cambridge Univ. Press.

SALAS, H. J. y P. MARTINO. 1990. Metodologías simplificadas para la evaluación de la eutrofización en lagos cálidos tropicales. CEPIS/HPE/OPS.

SCHEFFER, M., S. RINALDI, A. GRAGNANI, L. MUR Y E. VANNES. 1997. On the dominance of filamentous cyanobacteria in shallow turbid lakes. Ecology, 78: 272-282.

SCHIAFFINO, M. 1981. Campaña limnológica "Keratella I" en el Río Paraná medio. Rev. Asoc. Cienc. Nat. Litoral, 12: 140-147.

SOMMER, U. 1993. Disturbance-diversity relationships in two lakes of similar nutrient chemistry but contrasting disturbance regimes. Hydrobiol., 249: 59-65.

SOMMER, U., J. PADISAK, C. REYNOLDS y P. JUHASZ-NAGY. 1993. Hutchinson's heritage: the diversity - disturbance relationship in phytoplankton. Hydrobiol., 249-1-7.

STOERMER, E. y J. SMOL. 1999. The Diatoms Applications for the Environmental and Earth Sciences. Cambridge Univ. Press.

TUNDISI, J. G. 1990a. Distribuiçâo espacial, sequência temporal e ciclo sazonal do fitoplânc- ton em Reservatorios: Fatores limitantes e controladores. Rev. Brasil Biol., 50(4): 937-955.

TUNDISI, J. G. 1990b. Limnology and eutrophication of Barra Bonita Reservoir, S. Paulo State, Southern Brazil. Arch. Hydrobiol. Beih. Ergebn. Limnol., 33: 661-676.

TUNDISI, J. G. 1983. A review of basic ecological processes interactin with production and standingstock of phytoplankton in lakes and reservoirs in Brazil. Hydrobiol., 100: 223-243.

TUNDISI, J. G., T. MATSUMURA-TUNDISI, R. HENRY, O. ROCHA y K. HINO. 1988. Comparaçâo do estado trófico de 23 reservatórios do Estado de Sao Paulo: Eutrofizaçâo e manejo. En: Limnologia e Manejo de Reservatórios. V. 1, T. 1. J. G. Tundisi (ed.). Universidad de Sao Paulo.

VILA, I., I. BARENS y V. MONTECINO. 1987. Abundancia y distribución temporal del fitoplancton en el Embalse Rapel, Chile Central. Rev. Chilena de Hist. Nat., 60: 37-55.

ZALOCAR, Y. y E. VALLEJOS. 1982. Fitoplancton del Río Alto Paraná. Variación estacional y distribución en relación a factores ambientales. Ecosur, 9(17): 1-28. 